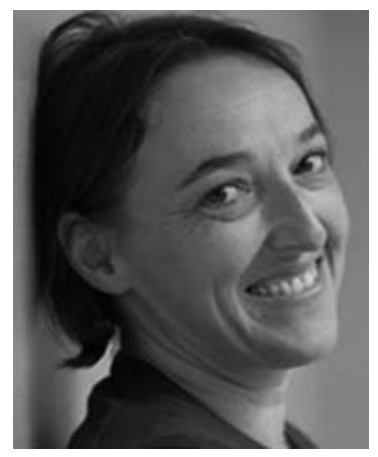

\title{
A Conversation with Angelika Amon
}

\author{
INTERVIEWER: Guy RIDDIHOUgh \\ Senior Editor, Science
}

Angelika Amon is a Professor at the Koch Institute for Integrative Cancer Biology, Massachusetts Institute of Technology.

Guy Riddihough: Tell us about chromosomes and cell division, and what happens so that we can understand why it's important to know how it works.

Dr. Amon: The DNA, which encodes all the information to build an organism, is basically strings of information. Before a cell divides, it looks like spaghetti soup. There's all these strings swimming around in that soup. If you want to make two cells, you need to copy the spaghetti and then divide them so that each daughter cell receives a copy of each spaghetti. To divide the spaghetti, you further need to compact them and then attach them to an apparatus that segregates them. This is an unbelievably complicated process, yet cells do it with such ease, and more importantly, with such accuracy. We want to understand how cells evolved to get it right virtually every single time. Ten trillion times to build you and me, starting from a single cell: a fertilized egg.

Guy Riddihough: How many chromosomes does a human cell have?

Dr. Amon: We have 46 chromosomes-23 pairs. You each get one set from your mother and one from your father. Obviously if the parents have two pairs of each, if you just put two of those cells together the next generation would have four copies, so you need to have a cell division that halves the chromosome content to make sperm and eggs that have half the amount of chromosomes: 23. When a sperm and egg fuse, you'll get back to the original 46 , and you can restart the cell division process. This division that generates sperm and eggs and results in the halving of chromosome number is called meiosis. We're trying to understand how the normal cell division-mitosis - that builds an organism from a single egg is modified so that you can bring about this highly unique cell division that gives rise to sperm and egg.

Meiosis also occurs with enormous accuracy, but it's not quite as accurate as mitotic cell divisions. Errors occur much more frequently. Many birth defects (e.g.,
Down Syndrome) are the result of an inaccurate cell division that gives rise to a sperm and an egg.

Guy Riddihough: So meiosis makes haploid germ cells - sperm and ova - that join up to make a diploid cell, and then that diploid cell goes through a whole series of mitoses where you simply copy the chromosome number each time.

You keep talking about accuracy, which is critical because each chromosome is unique and has its own set of specific genes that aren't found on other chromosomes. So any one chromosome is absolutely critical, because it will have genes necessary for the development of the organism.

Dr. Amon: Absolutely. For example, Down SyndromeTrisomy 21 - is a disease where a human being has three copies of chromosome 21 instead of two. That has a profound impact on the health and abilities of this individual. That's a terrific illustration for how important it is to segregate your chromosomes accurately. Of course, chromosome missegregation during the mitotic division that build the organism can also lead to diseases such as cancer. In fact, a key characteristic of cancer cells is that they have the wrong chromosome composition. They're losing and gaining bits and pieces of DNA, and that allows them to escape the controls that tell the cell to divide or not. That can lead to cells growing excessively and forming tumors or growing in places where they are not supposed to grow.

Another interest of my lab is to understand what happens to cells in which the normally accurate process of chromosome segregation fails, and what happens to cells that end up with the wrong chromosome composition. That's a condition known as aneuploidy, and it has a significant impact on human health. The literature also suggests that the degree of aneuploidy - the number of cells in a body that has the wrong chromosome numberincreases with age. Some researchers even suggest aneuploidy could actually be a cause of aging. 
Guy Riddihough: Aneuploidy is specifically when a cell has either gained or lost a whole chromosome, rather than just a losing a fraction of a chromosome. Can't the cell protect itself against that or kill itself if it's lost an entire chromosome?

Dr. Amon: Currently, we lack evidence that this is the case. Cells appear to optimize the process of dividing chromosomes to such a high fidelity that having a mechanism that eliminates cells with the wrong chromosome number afterward is perhaps not necessary. The processes that ensure that chromosomes are segregated accurately are called surveillance mechanisms, or checkpoints. Cells will only initiate chromosome segregation when they are absolutely sure that every one of the 46 chromosomes has accurately attached to the apparatus that segregates them correctly. Although chromosome missegregation events happen about once every 100,000 times, we have no evidence to suggest that they actually lead to a response where the cells that end up with the wrong number of chromosomes die or won't divide anymore. They are, however, a lot less fit. They have a lot of stresses that have been characterized, and if they continued to proliferate over time, they would lose out compared to normal cells that have the correct chromosome number. Once you've made a mistake, you're pretty much stuck.

Guy Riddihough: So the consequences are different depending on what type of cell division it is. In meiosis, the consequences of generating an aneuploid sperm or ova is that that particular germ cell forms the organism, and every single cell in that organism will have the incorrect chromosome number. Whereas mitosis is slightly different, because you could effectively have a chimera.

\section{Dr. Amon: Mosaicism.}

Guy Riddihough: That's when you have a small, potentially growing, population of cells in the body that have the wrong chromosome number. If you have an aneuploid cell in a population of wild-type cells, why isn't it eliminated?

Dr. Amon: If that aneuploid cell was a stem cell whose job it is to divide repeatedly over time to replenish a tissue, the contribution of progeny that the aneuploid stem cell would contribute to the overall soma would be less than that of a euploid stem cell, because these cells are less fit. They are stressed and proliferate less effectively. However, a terminally differentiated cell in a tissue that develops aneuploidy just sits there. It really doesn't have to do much other than basic housekeeping functions. In a terminally differentiated tissue, aneuploidy is tolerable to a certain degree. However, there's a rare disease in humans that causes an increased frequency in chromosome missegregation, and people affected with this disease have a lot of problems.

If the entire organism is aneuploid because the germ cell that created it was aneuploid, then the consequences are dire. Virtually all chromosomal aneuploidies are lethal. A fetus with the wrong chromosome number will not develop to term and will spontaneously abort during pregnancy. Often, women don't even know they are preg- nant. Sometimes, as in the case of Down Syndrome, these children survive to birth, but that is really very rare.

Guy Riddihough: What are the consequences of aneuploidy for the cell? Are there effects on transcription or gene expression?

Dr. Amon: Chromosome losses and gains directly impact gene expression. If you gain an extra chromosome, you will express all three genes instead of two. Chromosome gains are easier to deal with than losing a chromosome. When you gain a chromosome and produce extra RNAs and extra proteins, cells have some quality control capacity to eliminate these excesses, but it's not $100 \%$ successful. Over time, these extra proteins accumulate, misfold, or aggregate, and that causes problems for protein homeostasis.

On the other hand, losing a chromosome reduces gene expression because you now have only one copy instead of two. When you lose a chromosome, the consequences for the cell, for the tissue, for the organism, are much more dire because some processes are difficult or impossible to run on half the amount of gene dosage than what's normally necessary.

Guy Riddihough: Aneuploidy is a prominent characteristic of cancer. Is this an early driver of tumorigenesis, or is it a later process that turns an initial-phase cancer into something more dangerous?

Dr. Amon: Early malignant lesions or even premalignant lesions are already aneuploid, having a few chromosome gains or losses, although nothing drastic. This observation suggests that aneuploidy is an early event in tumorigenesis and could even drive the process. However, the experimental evidence is not as clear-cut. Mouse models that simulate increased chromosome gains or losses do not necessarily result in the animals developing cancer more readily, nor is the disease necessarily more aggressive. Sometimes it helps, sometimes it doesn't, and it seems to be very dependent on which tissue you're looking at. So the relationship between a wrong karyotype and cancer is not a simple, linear relationship.

Our own work suggests that aneuploidy adversely affects fitness and that the proliferative capabilities of all types of cells, normal and cancerous, are decreased by aneuploidy. We don't think that aneuploidy, per se, drives tumorigenesis, but that instead aneuploidy increases variability. Changes in gene dosage cause cells to have different characteristics that under some circumstances might be advantageous, though generally they are disadvantageous. Furthermore, aneuploidy causes other additional changes in the genome. It's what we call "mutagenic." It increases genomic instability and causes all forms of alterations of the genome. We believe that it's this ability to mutate the genome that is a driver of tumorigenesis.

Guy Riddihough: Does that mean that addressing aneuploidy in terms of medical treatment would be very difficult both in terms of women's reproductive issues, and also in terms of treating cancers or other diseases related to it? 
Dr. Amon: With respect to fertility treatments, the singlecell karyotype analysis that can now be performed in very early in vitro fertilized embryos has become so advanced that it's much more feasible to test the embryos and select one that has the right set of chromosomes before they are implanted into the womb.

In terms of cancer, we are exploring the possibility of aneuploidy as a therapeutic target because it's a universal characteristic of cancer, and because aneuploid cells have so many stresses and many types of aneuploidies share specific stresses. The pharmaceutical industry is pursuing personalized medicines targeting very specific mutations in specific types of cancer. I think we should also consider going after the more general and universal traits of the disease such as aneuploidy. I think they are equally viable targets, and they would have a much broader efficacy. We are actively looking for compounds that selectively inhibit the proliferation of aneuploid cells.

Guy Riddihough: On another subject, as a successful woman in science, what do you think needs to happen for more women to be successful, and for the women that are already in science to be more successful?

Dr. Amon: In my personal experience, raising the number of women in science at a particular institution and putting them into prominent positions makes it seem "normal." That's what I want to see: to not be the odd peacock out, the token woman who speaks at a meeting. If half the speakers are women, after 2 years people won't blink an eye anymore. I grew up in Austria, a very maledominated country. My grandfather was of the firm opinion that women, simply because they're women, are terrible car drivers. That's what I heard as a 5-year-old child, and I assumed women aren't very good drivers. I didn't question it. My grandfather said it every time I drove in the car with him.

Obviously, nowadays nobody in their right mind would think that. I think that's the same with everything. As long as you just increase the presence of underrepresented minorities, of women, in a particular field, in a particular job, in any particular environment, it'll become normality. The same is true for MIT. Until 1970, there were hardly any women students. Now $50 \%$ of those undergraduate students are women, and it's completely normal. My personal opinion is that it's important to conscientiously increase the presence of women in science at every level, especially at the level of professor and team leader. I'm a firm believer in affirmative action. It works.

Guy Riddihough: Does there need to be any special catering for women? Especially with regard to fitting in, having children with a career, or do you think that women and men can be considered equal on that?

Dr. Amon: There should be a 1-year extension of the tenure clock for every parent. In our times, men are much more involved in the rearing of the children than they ever have been. The child issue puts more pressure on women than men, simply because they have to bear them and then they have to feed them. Making it easier for young people to have children, irrespective of whether they're men or women, is a good thing. In science, we do a lot. Having day care or a liberal work schedule is much more common compared to other professions. It's very difficult for people who work in high-powered business or law firms to go home at three, spend a few hours with their child, put him or her to bed, and then come back to work. As scientists and academics, we're really blessed to have that liberty. I think making it better for all parents will only do good, irrespective of the sex of the parents. 


\section{$\$_{\text {CSH }}^{\infty}$ Cold Spring Harbor Symposia SYMPOSIA On Quantitative Biology}

\section{A Conversation with Angelika Amon}

Cold Spring Harb Symp Quant Biol 2015 80: 299-301

Access the most recent version at doi:10.1101/sqb.2015.80.029942

\section{License}

Email Alerting Receive free email alerts when new articles cite this article - sign up in Service the box at the top right corner of the article or click here. 